\title{
Implementation of Smart Manufacturing Maturity Assessment Framework: A Socio-Technical Perspective
}

\author{
Lei Yue ${ }^{1 *}$, Zhou Wang ${ }^{1}$, Yifang Fang ${ }^{1}$ and Zhonghua $\operatorname{Han}^{2,3}$ \\ ${ }^{1}$ Instrumentation Technology and Economy Institute (ITEI), Beijing 100055, China \\ ${ }^{2}$ Shenyang Institute of Automation, Chinese Academy of Sciences, Shenyang 110016, China \\ ${ }^{3}$ Faculty of Information and Control Engineering, Shenyang Jianzhu University, Shenyang 110016, China
}

\begin{abstract}
Smart manufacturing capability maturity assessment has been widely carried out in order to help enterprises to identify the direction and route of smart transformation by stationing in enterprises. However, many maturity models for industrial enterprises can not reflect their industrial realities more accurately. Therefore, the aim of this paper attempts to make the smart manufacturing maturity assessment operational, and implement a specific assessment framework based on an existing general conceptual framework. This implementation involved two top-down approaches that one is socio-technical system as a top-level design of factors, and the other is refining assessment domains based on lifecycle and activity models. Results of this implementation showed a two-dimensional assessment framework instance and collections of definitions for capability maturity levels. To conclude, this implementation may be a reference for work specification of smart manufacturing maturity assessment. Three possible future research in the area was suggested at last.
\end{abstract}

\section{Introduction}

Entrepreneurs are willing, but worried and confused, to develop Smart Manufacturing. The first problem is unclear about where to start when facing so many topics such as R\&D, production, management, logistics, sales. More recently, with an increase in capability maturity approaches to the assessment of enterprises, we have seen a shift in interests of research. Smart manufacturing capability maturity assessment has been widely carried out in order to help enterprises to identify the direction and route of smart transformation by stationing in enterprises. It has enabled enterprises to determine their position on the road of Smart Manufacturing transformation and upgrading.

Several studies (Schumacher, 2016; De Carolis, 2017) have compared existing maturity and readiness models in Smart Manufacturing or Industrie 4.0 domains[1, 2]. Mittal (2018) has critically reviewed currently available smart manufacturing or Industrie 4.0 maturity models, and provided insights that help towards developing a realistic Smart Manufacturing or Industrie 4.0 maturity model for enterprises that reflects their industrial realities more accurately[3]. Another paper in Mittal (2018) collects and structures the various characteristics, technologies and enabling factors available in the current body of knowledge that are associated with Smart

\footnotetext{
*Corresponding author's e-mail: yuelein@126.com
}

Manufacturing[4]. It can be concluded that most of the recent studies focus on the technical aspect of an enterprise. What could be likely to lose focus is that an enterprise often presents a social aspect because of the existence of human agency. The ultimate effectiveness of Smart Manufacturing or Industrie 4.0 is largely human agency-related.

In considering of both technical and social aspects of an enterprise, Yue (2019) proposed a rough general conceptual framework from a socio-technical perspective for understanding smart manufacturing maturity assessment in order to revise assessment elements so that the assessment results can truthfully reflect objective reality[5]. Essentially, the enterprises adopting smart manufacturing technologies is a socio-technical system (STS). In this paper we present an implementation of Smart Manufacturing Maturity Assessment Framework on basis of Yue (2019)'s study.

This implementation was guided by the following research questions: (a) How to construct a specific assessment framework on basis of the general conceptual framework, and (b) How to define smart manufacturing capability maturity level. Such implementaiton is still in its infancy, but it may have a contribution to subsequent empirical studies and questionnaire survey in specific assessing.

The remainder of this paper is divided into three sections. The Section 2 provides a brief introduction to 
taxonomy system derived from STS and activity models. Section 3 provides an instance of smart manufacutring assessment framework, and presents the definition collections for capability maturity level. Conclusions are presented and suggestions are made for further research in Section 4.

\section{Approach}

In Yue (2019)'s study, the assessment framework for smart manufacturing is a three-dimensional space which is composed of "Domains", "Factors" and "Visions". "Domains" represents the collections of activities performed in an enterprise. "Factors" represents the collections of smart technologies employed in an enterprise, management of these smart technologies, and human agencies of adopting them. In this paper, we extend the meaning of "Visions", and suggest that it represents the collections of stakeholder's particular concerns to be addressed and meanwhile implies the economic value that an enterprise pursues.

In this implementation, we propose the following two Top-down approaches respectively to "Factors" design and "Domains" identifying for instantiating the first two dimensions of Yue's general conceptual framework. While "Visions" will be classified into different ranks in terms of different stage theory for capturing capability maturity levels.

\subsection{Socio-technical system as a top-level design}

Social practices in industrial history have shown that it is not sufficient to design and select an industrial technical system with only an eye to the coordination and balance between technical and economic objectives. Decisionmaking in modern industrial enterprises should consider not only the technical-economic criterions, but also sociotechnical and ethic criterions.

Enid Munford's research revealed that when designing socio-technology system, the core concern is to find the best match and joint optimization of technology and social elements, so as to improve both economic performance and quality of work[6]. The explanation of the mechanism behind the interaction between human and technology has gone beyond the categories of sociology and management theory to the height of philosophical ontology. Orlikowski (2007) and Leonardi (2012) revealed the essence of technology using the term "sociomateriality" $[7,8]$.

The decisions made in a modern industrial enterprise could be separated into administrative level, economic level and technical level. Where, the technical level decisions are subject to and regulated by the economic level visions. While, the economic level visions are limited by the administrative level policies and strategies. Such a socio-economic-technical system is self-adaptive, and learns through decision making and practices. Its structure, capabilities, welfare, and ideology are continuously evolving to an advanced paradigm over time as soon as it embarks on a road of continuous improvement[9].

Yue's general conceptual framework used the sociotechnical system as the top-level design of the "Factors". On this basis, we use top-down approach to instantiating the abstract "Factors" and obtain specific applicable factors. The results will be shown in Section 3.

\subsection{Refining assessment domains based on lifecycle and activity models}

Four significant perspectives have been identified in Yue's research which are "Business", "Product", "Factory" and "Operations Management". In this implementation, we will break down "Product" and "Factory" in terms of their respective lifecycle phases, and unfold "Business" and "Operations Management" with respective involved activities.

To cope with the refining of the two perspectives "Business" and "Operations", we propose a conjunctive model as a top-down approach as illustrated in figure 1, which could encompass the business and operations activities of most industrial enterprises. 


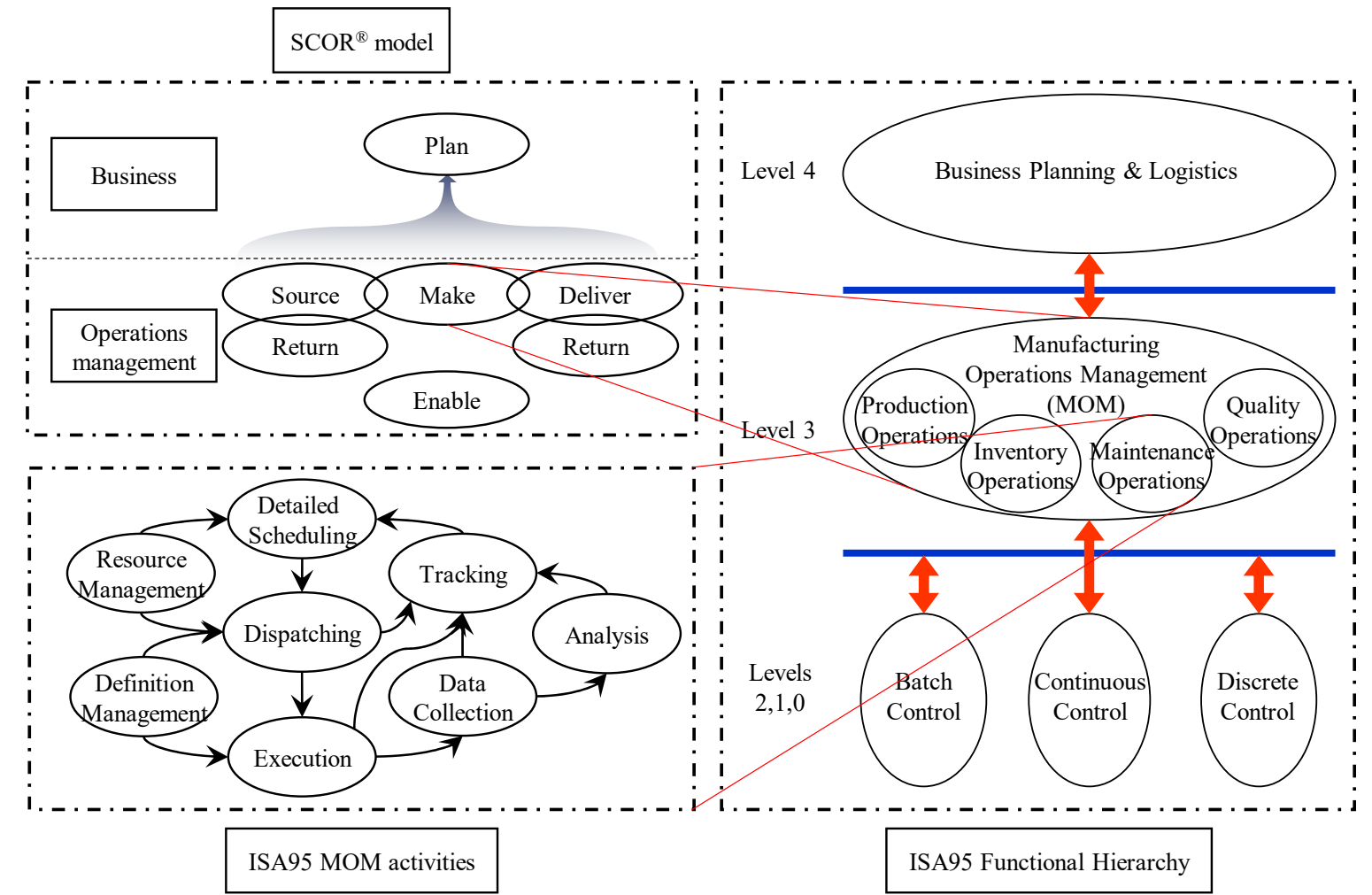

Figure 1. A conjunctive model aligning ISA95 model with $\mathrm{SCOR}^{\circledR}$ model

This conjunctive model employs the Supply Chain Operations Reference $\left(\mathrm{SCOR}^{\circledR}\right)$ model as the top-level design of "Business" and "Operations". The SCOR ${ }^{\circledR}$ model was developed in the early 1990s by consulting firm Pittiglio, Rabin, Todd \& McGrath (PRTM) and AMR Research with the assistance of individuals from corporations, to develop a methodology to improve supply chain performance. In 1996, PRTM and AMR founded a user-based organization, the Supply Chain Council (SCC). Starting with the original 69 corporations driving its inception. By 2012, the initial $\mathrm{SCOR}^{\circledR}$ model had progressed through 12 revisions. In 2014, SCC merged with American Production and Inventory Control Society (APICS). The $\mathrm{SCOR}^{\circledR}$ method was integrated into APICS's Operations Management Body of Knowledge framework to provide optimized supply chain management knowledge, best practices, and performance[10, 11].

$\mathrm{SCOR}^{\circledR}$ model provides a set of pre-defined descriptions for activities performed by most industrial enterprises to effectively execute their supply chains. There are six major processes have been recognized, which are "Source", "Make", "Deliver", "Return" and "Enable" in "Operations" layer, and "Plan" in "Business" layer. In this implementation, we employed six sub processes to unfold "Plan" and fill in the "Business" perspective. Next, we aligned Manufacturing Operations Management (MOM) activity models in ISA95[12] with "Make" process in $\mathrm{SCOR}^{\circledR}$ model. Therefore, it is possible to unfold the "Make" process in a top-down way with ISA95 MOM activities and obtain the next level processes. Moreover, we added an additional process "energy operations" into the "Operations Management" perspective which is taken no account of in ISA95 model.
On the other hand, we selected the most representative lifecycle phases to break down the other two perspectives "Product" and "Factory". These results will be shown in Section 3.

\section{Results}

In the progress of answering the two research questions raised in the introduction, we obtain the corresponding implementation results from two aspects. The first aspect is a specific assessment framework instance, and the second aspect is a series of discrete capability maturity levels.

In this section, we will present the results of these two aspects and provide concise explanations about them.

\subsection{A two-dimensional assessment framework instance}

The first aspect of our implementation results is the instantiation of the "Domains" and "Factors" dimensions. It is shown as a two-dimensional assessment framework in figure 2. We placed the "Domains" dimension and its contents horizontally and "Factors" dimension and its contents vertically. The intersection of each domain and factor denotes the collections of the capability maturity levels to be defined.

Taking a look at the horizontal lines along the vertical direction, in the "Technical subsystem" field, we listed the most common information systems, simulation systems, control systems, smart equipment and instrumentations, human skills and experience as known to most industrial enterprises according to the classification of "Cyber 
artifacts", "Physical artifacts" and "Human", but not limited to these listed factors. While, some specific management policies such as "5S management", "lean", "total quality management" (TQM), and "total productive maintenance" (TPM) were filled in "Socio subsystem" field. All of these are capability factors to be assessed.

By exploring the vertical lines along the horizontal direction, a series of detailed domains can be found, such as "Product Design", "Process Planning", "Process Engineering", and "Product Service" belonging to "Product" domain, and "Facilities Plan", "Logistics Plan", "Engineering \& Commissioning" involved in "Factory" domain.

Before the using of this two-dimensional assessment framework, the specific industrial sector or industrial enterprise should be determined first, so as to determine whether it makes sense to define the capability maturity level of a factor in a domain. We have an example of the pharmaceutical industry in figure 2. The intersection of two dimensions was coloured in grey if the artifact or agency in horizontal line does make sense to the domain in vertical line, otherwise, it was filled in with a cross. For example, some information systems such as CAD, CAE, CAPP, and CAM are not suitable for pharmaceutical industry. In addition to these two cases, there are plenty of intersections of a third case that is left blank which indicate an uncertain state. That is to say, whether the technical artifact or human agency can be applied to the domain is not yet certain.

Defining such an assessment framework is the basis for carrying out the activities of identifying the "as-is" levels of capability factors and diagnosing problems.

\subsection{Collections of definitions for capability maturity levels}

The second aspect of our implementation results is collections of definitions for capability maturity levels. Here, we suggest there are a family of capability maturity classes. We have identified at least three significant capability maturity classes, which are capability maturity for management, capability maturity for technology, and capability maturity for technology-task fit (TTF). Furthermore, the capability maturity for technology is classified into two subclasses, which are capability maturity for cyber artifacts and capability maturity for physical artifacts. Because the capability maturity levels for 


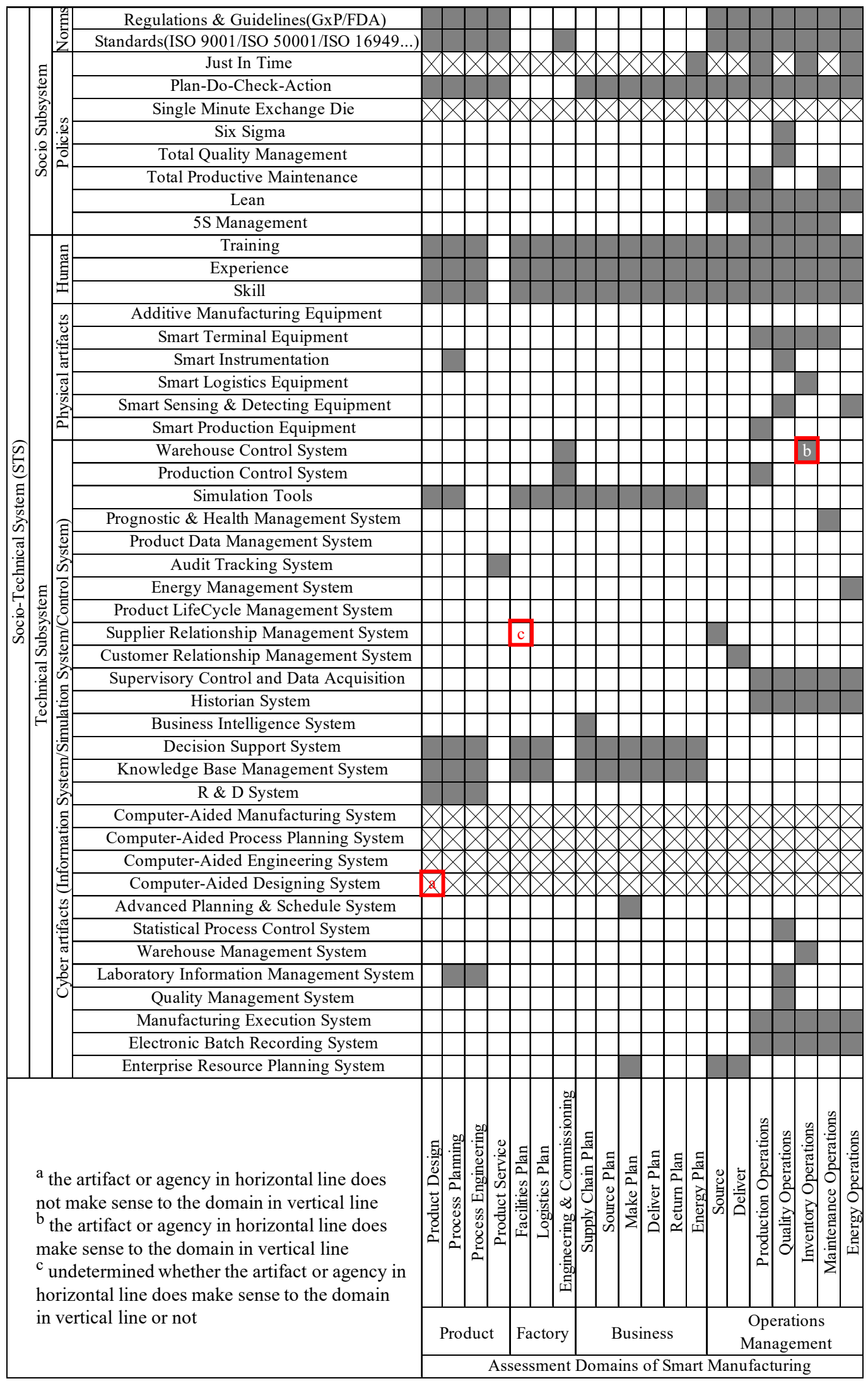

Figure 2. A two-dimensional assessment framework instance.

technology-task fit are still in developing, we just present the first two capability maturity classes in this paper.
There have been a lot of researches on management maturity. We provide one of the popular definitions as shown in figure 3(a). The following definitions determine 
the characteristics for a level of a factor in the socio subsystem field and an activity in the domain.

Definitions of capability maturity levels for management:

- Undefined: no related activities performed complying with the corresponding polices.

- Initial: the activities performed complying with the corresponding policies are undocumented, or not formally managed, and in a state of dynamic change, tending to be driven in an ad-hoc, uncontrolled and reactive.

- Managed: some of the activities performed complying with the corresponding policies are repeatable, possibly with consistent results. Not all activities are documented with consistent quality and detail. Activities vary across organizational groups, with different processes and procedures for similar tasks used in different groups.

- Defined: all the activities performed complying with the corresponding policies are defined with documented standards. Activities are defined across all organizational groups, and the organization follows written policies. Subgroups tailor their activities from organization standards.

- Quantitatively Managed: all the activities performed complying with the corresponding policies are defined, repeatable, monitored and managed across all organizational groups using agreed to metrics to manage the effectively.

- Optimizing: all the activities performed complying with the corresponding policies focus on continually improving performance. Key Performance Indicators (KPIs) on activities are collected and reviewed to discover problems.

Our perspective on the capability maturity for cyber artifacts is subject to the influence of theory of Technology Acceptance Model (TAM). Therefore we introduce an independent dimension "Technology Acceptance" that is orthogonal to "Information processing" to the capability maturity model of cyber artifacts in order to revise our assessment results of cyber artifacts (see figure 3(b)).

The "Information processing" dimension reflects the intelligence degree of the cyber artifacts from an epistemology angle. While, the "technology acceptance" reflects the acceptance degree of the cyber artifacts from an axiology angle.
Definitions of capability maturity levels for information processing are as follows:

- Default: no information processing capability.

- Data: collect data during the execution of transactions, keep records of these transactions, and may involve some simple mathematical operations on the data as required by high-level systems, but there is no contextual aggregation of data.

- Information: aggregate data in context, and provide structured information that allows managers to compare current and previous outputs for assessing the performance of the organization.

- Knowledge: usually with a knowledge base (or case library, model library), provide senior managers with analysis tools through "what-if" analysis and interactive question-and-answer method. Recommend solutions to managers but do not replace people to make decisions.

- Wisdom: deal with semi-structured and unstructured issues, to help executives analyzing the business environment, predicting long-term trends and situations. Recommend appropriate action plans.

Definitions of capability maturity levels for technology acceptance are as follows:

- Initial: initiate investments in the cyber artifacts, and acquires simple information systems/control systems/simulation tools which can be deployed in isolated environments.

- Recognition: recognize the importance and competitiveness of cyber artifacts and incorporate the planning, acquisition, development and maintenance of the cyber artifacts as part of regular business processes.

- Diffusion: diffuse the coverage of cyber artifacts throughout all value chains of the enterprise, and begin to obtain the support of the business departments.

- Control: control enterprise-wise cyber artifacts and increase their utilization and satisfaction through regular planning, documentation, and communication.

- Integration: integrate all value chains of the enterprise via cyber artifacts, and performance is fully determined by the data and functions provided by the cyber artifacts.

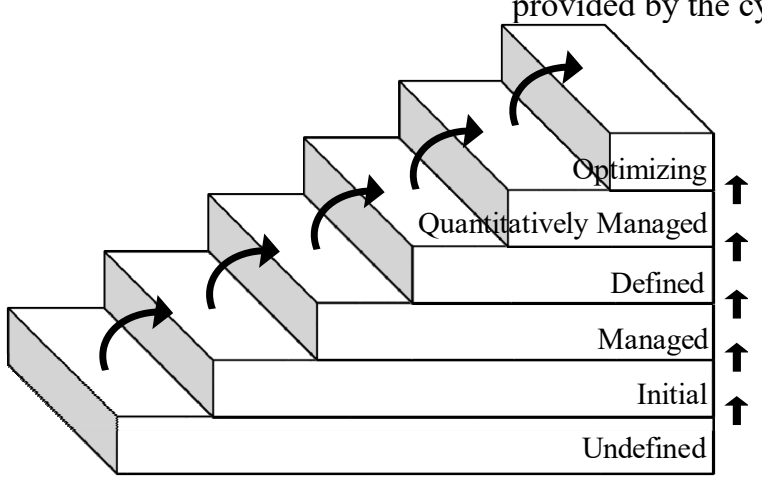

(a). Capability maturity for management 


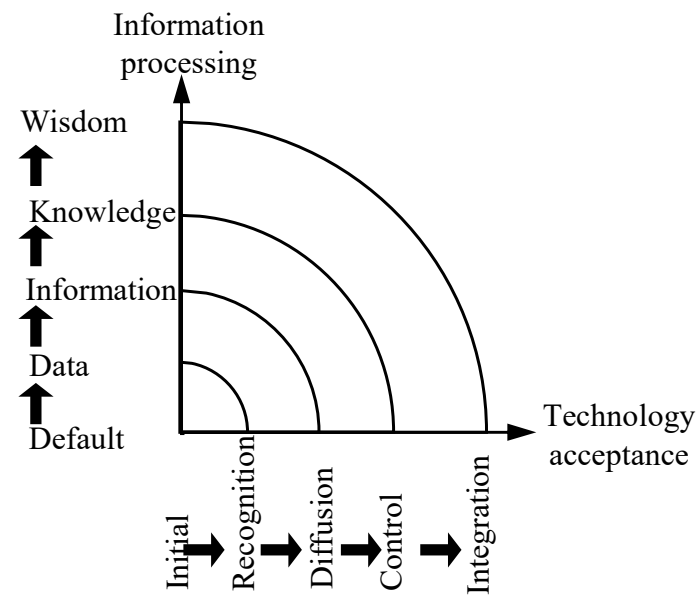

(b). Capability maturity for Cyber artifacts

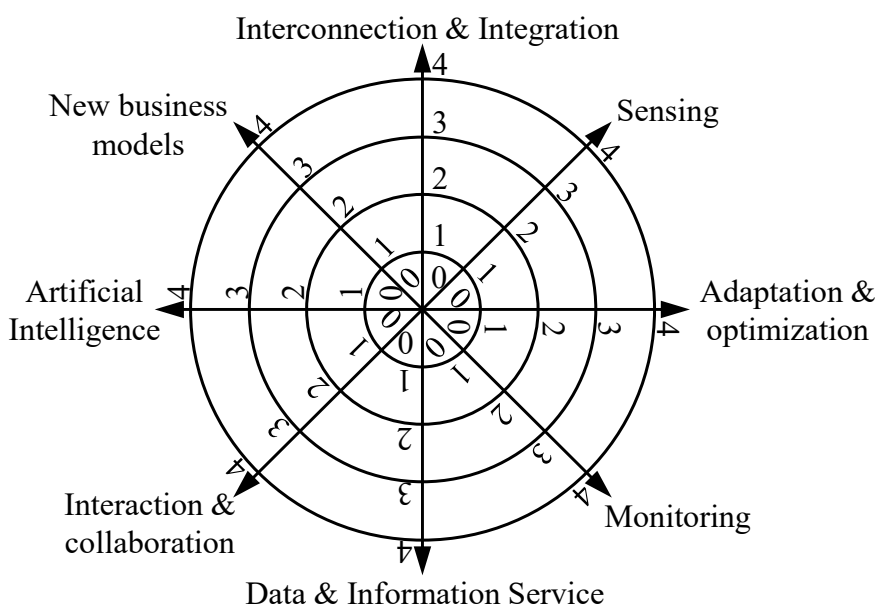

(c). Capability maturity for Physical artifacts

Figure 3. A family of capability maturity class

The capability maturity for physical artifacts is broken down into eight dimensions, which are interconnection \& integration, sensing, adaptation \& optimization, monitoring, data \& information service, interaction \& collaboration, artificial intelligence, and new business models (see figure 3(c)).

Definitions of capability maturity levels for physical artifacts are as follows:

\section{Interconnection \& integration}

- Level 0: isolated from each other, unable to interconnect and integrate functions.

- Level 1: coexist with communication protocol.

- Level 2: data interconnection and integration

- Level 3: business interconnection and integration

- Level 4: interoperability

Sensing

- Level 0: unable to collect data.

- Level 1: collect data and process information manually.

- Level 2: semi-automatic data collection and information processing.

- Level 3: automatic data collection and information processing.

- Level 4: information processing based on cloud platform.

Adaptation \& optimization

- Level 0: unable to adapt and optimize.

- Level 1: adjustment and optimization manually.

- Level 2: hard-coding, predetermined adaptation and optimization.

- Level 3: opening and automatic adaptation and optimization.

- Level 4: predictive, autonomous adaptation and optimization.

Monitoring

- Level 0: no monitoring, no interface for external monitoring or maintenance diagnosis information.

- Level 1: manual way to complete the monitoring of equipment, fault diagnosis and maintenance, etc.
- Level 2: monitoring and diagnosis of predetermined response patterns.

- Level 3: automatic monitoring and diagnosis.

- Level 4: predictive monitoring and diagnosis.

Data \& information service

- Level 0: independent, no data service.

- Level 1: provide its basic information, such as specifications, instructions for use, packaging instructions, etc.

- Level 2: provide information about the use of specific functions, or information generated in its operations.

- Level 3: provide information about the operations of itself and related systems, or information generated by itself and related systems.

- Level 4: provide information about the operations of itself and systems collaborating with each other, or information throughout its entire lifecycle.

Interaction \& collaboration

- Level 0: no human-machine interaction, no information feedback.

- Level 1: only provide information displaying, no operative functions.

- Level 2: provide information displaying, and manual operative functions.

- Level 3: provide multi-interaction channel information displaying, and carry out some intelligent assistant operations.

- Level 4: fully automatic identifying, understanding of the operator's intention according to the scenarios, to achieve intelligent assistant operations.

\section{Artificial intelligence}

- Level 0: no artificial intelligence.

- Level 1: limited to some predetermined scenarios and functions, unable to solve problems by independent reasoning.

- Level 2: partial reasoning in some predetermined scenarios. 
- Level 3: reasoning in opening scenarios.

- Level 4: reasoning highly consistent with human beings.

\section{New business models}

- Level 0: no emerging business models.

- Level 1: plan to establish emerging business models based on equipment.

- Level 2: emerging business models based on equipment are still in developing.

- Level 3: emerging business models based on equipment have been well constructed and are operating well.

- Level 4: emerging business models based on equipment is in the leading position in the peer.

\section{Discussions and Conclusions}

In this paper, we present the results of an implementation for smart manufacturing maturity assessment framework from a socio-technical perspective. These results provide a reference for the work specification of smart manufacturing assessment. Three of these implementation results are worth summarizing.

First, a large number of studies show that it is not the technology itself that affects the performance and benefits of enterprises, but the fit degree of technology and task, namely, the degree of technology assisting individuals to perform tasks. In other words, if enterprises would like to benefit from technology investments, they must from a benign interaction among tasks, technologies and individuals. Therefore, the evaluation of technology-task fit degree should be included in the assessment framework and the maturity levels for technology-task fit shall be defined. Future work will hopefully clarify this important concern based on the existing TTF theory.

Second, the construction of smart manufacturing assessment framework is of great significance not only in theory but also in practice. However, in many cases, the results of theoretical construction may not always conform to the laws of nature or social movement. The research paradigm of this paper belongs to theoretical research, which is also the shortcoming. Additional empirical research is needed on application effects of the assessment framework.

Third, we intend to design questionnaires based on the capability maturity models, and conduct several interviews with diverse industrial enterprises over the questionnaires for the purpose of eliciting industrial enterprises' requirements deeply.

\section{Acknowledgments}

This research was fully supported by a grant from the National key research and development Project "The research and development of the new framework for instrumentation supporting Semantic integration" (Grant No. 2018YFB2003200).

\section{References}

1. Schumacher, A., Erol, S. \& Sihn, W. (2016). A Maturity Model for Assessing Industry 4.0 Readiness and Maturity of Manufacturing Enterprises. Procedia CIRP, 52, 161-166.

2. De Carolis, A., Macchi, M., Kulvatunyou, B., Brundage, M. P. \& Terzi, S. (2017) Maturity Models and Tools for Enabling Smart Manufacturing Systems: Comparison and Reflections for Future Developments. In: RíOS, J., BERNARD, A., BOURAS, A. \& FOUFOU, S., eds. Product Lifecycle Management and the Industry of the Future, 2017// 2017 Cham. : Springer International Publishing, 23-35.

3. Mittal, S., Khan, M. A., Romero, D. \& Wuest, T. (2018). A critical review of smart manufacturing \& Industry 4.0 maturity models: Implications for small and medium-sized enterprises (SMEs). Journal of Manufacturing Systems, 49, 194-214.

4. Mittal, S., Khan, M., Romero, D. \& Wuest, T. (2017). Smart Manufacturing: Characteristics, Technologies and Enabling Factors.

5. Yue, L., Niu, P., Fang, Y. \& Han, Z. (2019). Towards a Smart Manufacturing Maturity Assessment Framework: A Socio-Technical Perspective. Journal of Physics: Conference Series, 1345, 042063.

6. Avison, D., Bjørn-Andersen, N., Coakes, E., Davis, G. B., Earl, M. J., Elbanna, A., Fitzgerald, G., Galliers, R. D., Hirschheim, R., Iivari, J., Klein, H. K., Land, F., De Marco, M., Pettigrew, A. M., Porra, J., Stahl, B. C., Sørensen, C., Wood, B. \& WoodHarper, T. (2006). Enid Mumford: a tribute. Information Systems Journal, 16(4), 343-382.

7. Orlikowski, W. J. (2007). Sociomaterial Practices: Exploring Technology at Work. Organization Studies: An international multidisciplinary journal devoted to the Studies of organizations, organizing, and the organized in and between societies $\$ V 28$, (9), 1435-1448.

8. Leonardi, P. M. (2012). Materiality, Sociomateriality, and Socio-Technical Systems: What Do These Terms Mean? How Are They Related? Do We Need Them? In: LEONARDI, P. M., NARDI, B. A. \& KALLINIKOS, J. (eds.) Materiality and Organizing: Social Interaction in a Technological World. Oxford: Oxford University Press.

9. Zhu, S. \& Chen, T. (1996). A Survey on Multiobjective Comprehensive Assessment in Social Technology Systems. Systems Engineering, (04), 3539.

10. Zhou, H., Benton Jr, W. C., Schilling, D. A. \& Milligan, G. W. (2011). Supply Chain Integration and the SCOR Model. Journal of Business Logistics, 32(4), 332-344.

11. Cavusoglu, T., Gulledge, T. \& Kessler, T. (2001) Aligning the supply chain operations reference (SCOR) model with enterprise applications. PICMET '01. Portland International Conference on Management of Engineering and Technology. Proceedings Vol.1: Book of Summaries (IEEE Cat. 
No.01CH37199), 29 July-2 Aug. 2001 2001. : , 462 vol.1.
12. ANSI (2005). ANSI/ISA-95.00.03-2005 EnterpriseControl System Integration Part 3: Activity Models of Manufacturing Operations Management. 